\title{
REVIEW
}

\section{Meningococcal Vaccinations}

\author{
Nancy Crum-Cianflone $\cdot$ Eva Sullivan
}

Received: February 25, 2016/Published online: April 16, 2016

(C) The Author(s) 2016. This article is published with open access at Springerlink.com

\section{ABSTRACT}

Neisseria meningitidis, a gram-negative diplococcal bacterium, is a common asymptomatic nasopharyngeal colonizer that may infrequently lead to invasive disease in the form of meningitis or bacteremia. Six serogroups (A, B, C, W, X and Y) are responsible for the majority of invasive infections. Increased risk of disease occurs in specific population groups including infants, adolescents, those with asplenia or complement deficiencies, and those residing in crowded living conditions such as in college dormitories. The incidence of invasive meningococcal disease varies geographically with some countries (e.g., in the African

Enhanced content To view enhanced content for this article, go to http://www.medengine.com/Redeem/ DB84F0607E9D292D.

N. Crum-Cianflone $(\bowtie) \cdot$ E. Sullivan

Infectious Diseases, Scripps Mercy Hospital, San

Diego, CA, USA

e-mail: nancy32red@yahoo.com;

crum-cianflone.nancy@scrippshealth.org

N. Crum-Cianflone

Naval Medical Center San Diego, San Diego, CA, USA meningitis belt) having both high endemic disease rates and ongoing epidemics, with annual rates reaching 1000 cases per 100,000 persons. Given the significant morbidity and mortality associated with meningococcal disease, it remains a major global health threat best prevented by vaccination. Several countries have implemented vaccination programs with the selection of specific vaccine(s) based on locally prevalent serogroup(s) of N. meningitidis and targeting population groups at highest risk. Polysaccharide meningococcal vaccines became available over 40 years ago, but are limited by their inability to produce immunologic memory responses, poor immunogenicity in infants/children, hyporesponsiveness after repeated doses, and lack of efficacy against nasopharyngeal carriage. In 1999, the first meningococcal conjugate vaccines were introduced and have been successful in overcoming many of the shortcomings of polysaccharide vaccines. The implementation of meningococcal conjugate vaccination programs in many areas of the world (including the massive campaign in sub-Saharan Africa using a serogroup A conjugate vaccine) has led to dramatic 
reductions in the incidence of meningococcal disease by both individual and population protection. Progressive advances in vaccinology have led to the recent licensure of two effective vaccines against serogroup B [MenB-4C (Bexsero) and MenB-FHbp (Trumenba)]. Overall, the evolution of novel meningococcal vaccines and the effective implementation of targeted vaccination programs has led to a substantial decrease in the burden of disease worldwide representing a major public health accomplishment.

Keywords: Meningococcal;

Neisseria

meningitidis; Review; Vaccinations

\section{MENINGOCOCCAL DISEASE}

Meningococcal disease is caused by the gram-negative diplococcus, Neisseria meningitidis. Although clinical descriptions of the disease occurred in the early 1880s [1], the first description of the organism in cerebrospinal fluid (CSF) is attributed to Marchiafava and Celli [2]. Three years later, Weichselbaum identified and cultured the organism [3].

The meningococcus, which only infects humans, may colonize the mucosal surfaces of the nasopharynx and upper respiratory tract. Colonization occurs in approximately $10 \%$ (range 3-25\%) of the population with the highest rates among adolescents and closed populations (college students, military recruits and multi-person households) [4]. Colonization may last days (transient) or months (persistent), the latter of which may serve as an immunizing event providing protection against future invasive infection [5-7]. Acquisition of the bacteria from a carrier or infected person occurs through direct contact with large-droplet respiratory secretions, including activities such as kissing, coughing and sharing eating/drinking utensils.

Meningococcal disease develops after the bacteria invades across the mucosal surface and enters the bloodstream. Onset is typically rapid, and fulminant disease may develop 1-14 days after acquisition [8, 9]. Clinical syndromes include meningitis with or without meningococcemia, the latter often manifesting with a petechial rash or purpura fulminans [1]. $N$. meningitidis remains the first or second most common cause of meningitis in many geographic areas including the US and is the only bacterium with the ability to produce large meningitis outbreaks [10-12]. Other clinical presentations of $N$. meningitidis infection include pneumonia, arthritis, myocarditis, pericarditis, urethritis and endophthalmitis [1, $9,13,14]$.

Infections frequently occur among previously healthy persons often with resultant high morbidity and mortality. In the developed world, the mortality rate remains $10-15 \%$, with rates of $\geq 20 \%$ in developing countries [1]. Among survivors, up to $19 \%$ have long-term sequelae including neurologic disabilities, seizures, hearing or visual loss, cognitive impairment, and loss of digits or limbs $[8,9,15,16]$.

Given the ability of the meningococcus to cause rapidly fatal and epidemic disease worldwide, understanding preventive strategies against this pathogen represents a global health priority for healthcare providers, public health personnel, and government officials worldwide. This review article provides a summary of the epidemiologic global trends and risk factors of invasive meningococcal disease. Current preventive strategies are reviewed including the available meningococcal vaccines and vaccination guidelines. Finally, a concise 
summary of post-exposure prophylaxis and treatment are provided. The data within this article are based on previously conducted studies and do not involve any new studies of human or animal subjects performed by any of the authors.

\section{EPIDEMIOLOGY AND BURDEN OF DISEASE}

Meningococcal disease occurs worldwide with epidemiologic trends and causative $N$. meningitidis serogroups/sequence types (ST) varying widely by geographic location (Fig. 1) $[1,17]$. Serogroups of $N$. meningitidis are based on the polysaccharide capsular structure, with six $(\mathrm{A}, \mathrm{B}, \mathrm{C}, \mathrm{W}, \mathrm{X}$ and $\mathrm{Y})$ of the 12 known serogroups (A, B, C, E, H, I, K, L, W, X, Y and Z) causing the majority of disease [1, 18-20]. Specifically, the most common ST and clonal complexes (which are based on polymorphisms in seven housekeeping genes) include ST-5 and
ST-7 (capsular serogroup A); ST-41/44, ST-32, ST-18, ST-269, ST-8 and ST-35 (serogroup B); ST-11 (serogroups C or W); ST-23 and ST-167 (serogroup Y); and ST-181 (serogroup X). Of note, serogroup $\mathrm{X}$ accounts for only a small number of invasive meningococcal disease worldwide.

In Sub-Saharan Africa, A (ST-5 and ST-7) historically was the predominant serogroup, with W (ST-11), C and X (ST-181) causing smaller numbers of cases. Since the introduction of massive vaccination campaigns against serogroup A, meningococcal strains causing invasive disease have shifted towards non-A serogroups. In the US and Canada, serogroups B (ST-41/44, ST-32), C (ST-11) and Y (ST-23) are the major causes of disease, and in Europe serogroups B (ST-41/44, ST-32, ST-18, ST-269, ST-8) and C (ST-11) predominate, the latter showing dramatic declines after serogroup C conjugate vaccine campaigns were introduced [21-23]. Some European countries, such as those

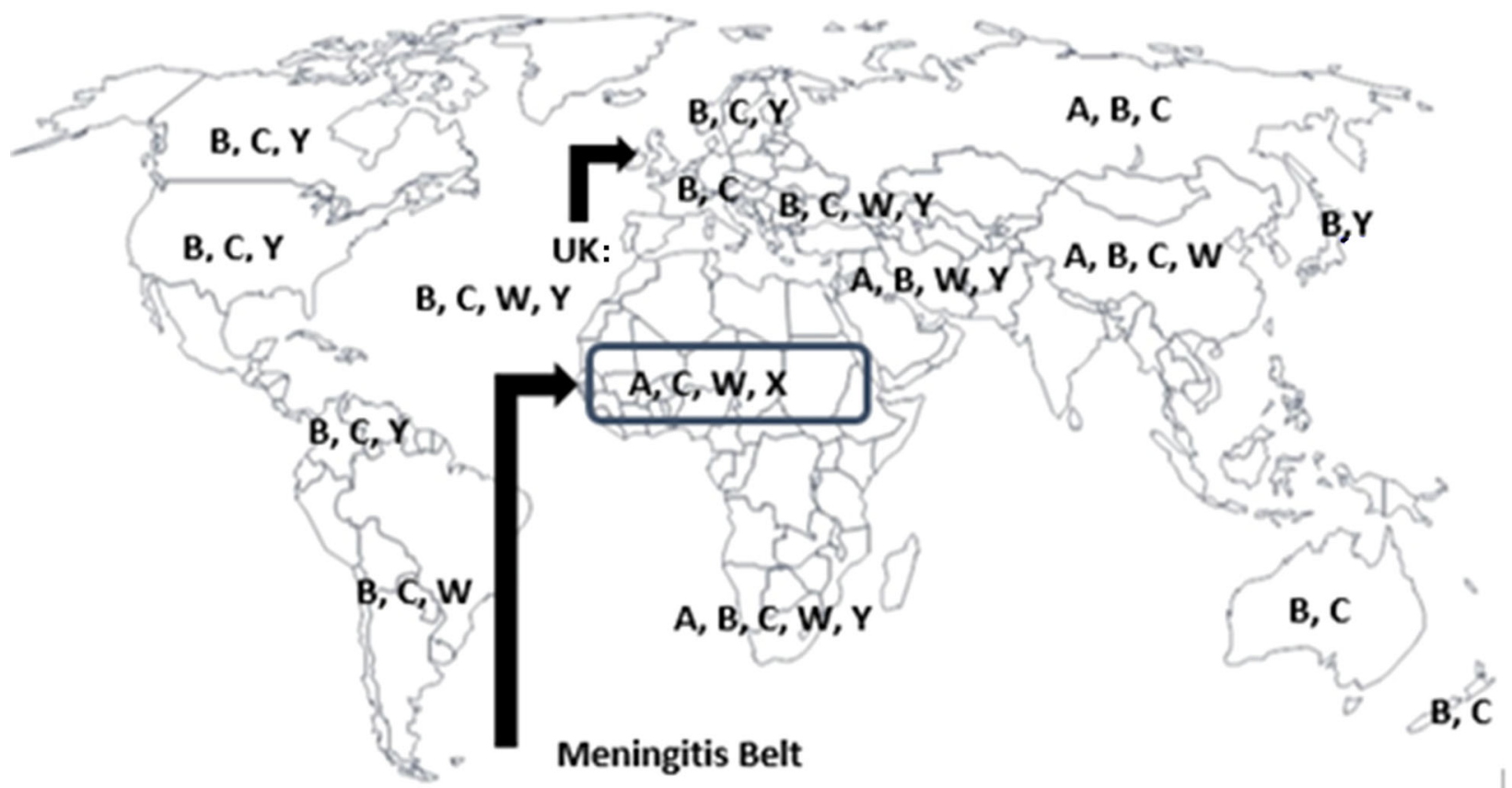

Fig. 1 Map of the predominant Neisseria meningitidis serogroups by geographic location 
in Nordic Europe, also are experiencing increasing disease from serogroup Y [24]. Further, a rapid expansion of serogroup W cases belonging to a single clone [sequence type 11 complex (cc11)] have recently emerged in England and Wales [25]. Serogroup B predominates in Australia and New Zealand with a notable reduction in serogroup $C$ post introduction of meningococcal $\mathrm{C}$ conjugate vaccine in Australia, whereas the incidence of serogroup $C$ has remained unchanged in New Zealand with approximately 10-30 cases per year during the past 10 years [22]. Serogroups A, $\mathrm{B}$ and $\mathrm{C}$ predominate in Russia; and based on limited data, serogroups $\mathrm{A}$ and $\mathrm{C}$ in Asia with serogroup B responsible for sporadic cases in China $[17,23]$. Serogroups B and Y cause the majority of meningococcal disease cases in Japan, even though the incidence is very low with an estimated $<30$ cases per year [17]. In South and Latin America, B and C are most common, with serogroup $\mathrm{Y}$ recently arising in Venezuela and Columbia and the incidence of serogroup $\mathrm{W}$ increasing in various countries in the Southern Cone in Latin America, a geographic region composed of the southernmost regions of South America, south of the Tropic of Capricorn, including Argentina, Southern Brazil, Chile, Paraguay and Uruguay [17]. In the Middle East and North Africa serogroups include $\mathrm{W}, \mathrm{B}, \mathrm{A}$ and $\mathrm{Y}$ [22, 26-28]. Finally, serogroup W (cc11) has also recently been increasingly reported in a wide diversity of locations worldwide including South America, South Africa and China [29-31].

An estimated $>1.2$ million cases and $>135,000$ deaths occur annually, with figures varying considerably given the natural peaks and troughs of the disease that occur every 5-12 years $[22,24,26-28]$. The epidemiology of meningococcal disease is determined by factors including population characteristics (e.g., age, presence of immunodeficiency), environmental conditions, presence of meningococcal serogroups that cause disease, and availability of medical therapies (e.g., antibiotics, vaccines).

Meningococcal disease can be epidemic, hyperendemic, or endemic (sporadic). For example, in the African meningitis belt, which is considered to have the highest incidence rates in the world, many cases are linked to large epidemics, whereas in the US, most cases (98\%) are sporadic, although outbreaks have been reported on college campuses [32, 33]. Large and devastating epidemics have plagued Africa as exemplified by the 1996-1997 epidemic resulting in $>250,000$ cases and $>25,000$ deaths [23]. While the incidence in Africa varies over time and by geographic location, annual rates up to 1000 cases per 100,000 population (which is 1 case per 100 persons) have been described [14, 22]. In Europe, the incidence is estimated as 0.92 cases per 100,000 population, while in the Americas rates range from 0.3 to 4 cases per 100,000 population [14, 22]. An estimated 600-1200 cases occur in the US annually with a current rate of 0.18 cases per 100,000 population $[34,35]$.

\section{RISK FACTORS FOR INVASIVE DISEASE}

Since less than $1-5 \%$ of persons exposed to the organism develop invasive disease [1], it is known that characteristics of the organism, host, and environment likely play a role in disease pathogenesis. Regarding the organism, capsular strains of $N$. meningitidis are more likely to cause invasive disease as the polysaccharide capsule serves as an important virulence factor allowing for evasion of opsonization as well as complement and phagocytic-mediated killing 
[36]. Further, specific genotypes of encapsulated organisms, designated as ST clonal complexes, vary in their virulence as shown by case:carrier ratio studies [7, 37]. For example, serogroup W (cc11) has been associated with both severe and unusual presentations of the disease [25, 29].

Host factors that result in the absence of protective bactericidal activity (due to a deficiency in complement or antibody production) are also risk factors for invasive meningococcal disease. These include functional or anatomic asplenia as well as persistent complement deficiencies (i.e., C3, C5-9, properdin, factor $\mathrm{D}$ and factor $\mathrm{H}$ ) [38, 39]. For example, terminal complement deficiencies (C5-9) are associated with a 1400to 10,000 -fold increase in meningococcal disease as well as the propensity for recurrent infections (in up to $50 \%$ of patients) $[1,38]$. Conditions with reduced complement levels such as systemic lupus erythematous (e.g., low C3 levels) also increase the risk. Similarly, medications that inhibit relevant host immune responses (e.g., eculizumab, a humanized monoclonal antibody that inhibits terminal complement) are risk factors [40].

Conditions associated with poor antibody responses (e.g., congenital or acquired antibody deficiencies such as seen in hypogammaglobulinemia and glomerulonephritis) also pose a risk for invasive disease $[5,6,41]$, and among these groups, atypical presentations of invasive disease have been described [41]. Whether HIV-infection is a risk factor has been debated; however, studies have shown those with AIDS or low CD4 counts are at higher risk compared with the general population [42]. Additionally, outbreaks have been described among HIV-infected men who have sex with men (MSM) engaging in high-risk sexual behaviors [43]. Finally, specific host genetic polymorphisms have also been associated with invasive disease [44-46] as well as extremes of age (e.g., $<6$ months and $\geq 65$ years) $[34,47]$.

Environmental factors that increase exposure to the organism include crowding and close contact with a person carrying the organism. For example, living in a residence hall during the first year of college has been associated with a higher risk of disease compared to non-college students of similar age groups [48]. As such, some countries (e.g., US) mandate education regarding meningococcal vaccinations among matriculating college students [49]. Other groups that may be affected by crowding include military recruits, with some militaries mandating vaccination against meningococcal disease at enlistment. Additionally, environmental exposures may be encountered by specific occupations such as microbiologists [50]; healthcare personnel in general are not felt to be at higher risk unless exposed to a patient with meningococcal disease [49]. Other environmental risks include residence or travel to hyperendemic areas (e.g., the meningitis belt in Africa) as well as pilgrimages to the Hajj in Mecca [51, 52]. Finally, antecedent respiratory infections (e.g., Mycoplasma, influenza and other viruses), exposure to active or passive smoking, and dry or windy air conditions are associated with a heightened risk for invasive disease due to mucosal damage and/or irritation [53-55].

An example of the culmination of multiple risk factors that previously led to extremely high incidence rates was seen in the African meningitis belt, stretching from Senegal in the west to Ethiopia in the east. During the dry season (December-June), dusty winds, cold nights and upper respiratory tract infections combined to damage the nasopharyngeal mucosa $[55,56]$. Additionally, overcrowding, 
exposures during migration and congregating at traditional markets, and underlying medical conditions enhanced the risk for disease. The combination of these factors and the presence of encapsulated, virulent serogroups of $N$. meningitidis explained the previous enormous burden of meningococcal disease observed in these areas prior to the institution of massive vaccination campaigns.

\section{MENINGOCOCCAL VACCINATIONS}

\section{Overview}

While $N$. meningitidis remains a formidable pathogen, tremendous strides toward its control have been made, including the development of effective vaccines, which first became available $>40$ years ago $[57,58]$. Advances in the immunogenicity and breadth of serogroup coverage of the vaccines have readily expanded over time [59].

\section{Available Vaccines}

Both polysaccharide and conjugated polysaccharide meningococcal vaccines are currently available (Table 1). Polysaccharide vaccines include bivalent (groups $\mathrm{A}$ and $\mathrm{C}$ ), trivalent (groups $\mathrm{A}, \mathrm{C}$ and $\mathrm{W}$ ) or quadrivalent (groups A, C, Y and W) forms. While initially vaccines were polysaccharide in nature, newer conjugate vaccines in which proteins are conjugated to polysaccharide antigens have been developed and available since the late 1990s. Polysaccharide vaccines are $T$ cell-independent and rely on humoral responses; hence, the immune protection may be short-lived. In contrast, conjugate vaccines generate T-cell-dependent antigen-recruitment responses. Advantages of conjugate vaccines include their ability to elicit immunologic memory; reduction in nasopharyngeal carriage and hence interruption of transmission and establishment of population protection; and effectiveness in protecting young children (<24 months) who may respond poorly to conventional polysaccharide vaccines. Available meningococcal conjugate vaccines include monovalent (A or C), quadrivalent (A, C, Y and $\mathrm{W})$ and combination (serogroup $\mathrm{C}$ or $\mathrm{C}$ and $\mathrm{Y}$, along with Haemophilus influenzae b) vaccines. No vaccine currently exists against serogroup X; however, candidate glycoconjugate vaccines are in development against this serogroup [60].

The development of an effective vaccine against serogroup B has been challenging, especially given the serogroup B polysaccharide resembles a human neural cell adhesion molecule, raising concern about autoimmunity [61]. Initially serogroup B vaccines were developed based on outer membrane vesicles (OMV) that contain proteins. OMV vaccines have been utilized in many areas (e.g., Cuba, South America, Norway and New Zealand) with demonstrated protective capacities against serogroup B. For example, the MeNZB ${ }^{\circledR}$, utilized in New Zealand, was effective in reducing rates of disease and controlling a serogroup B epidemic [62]. The main limitation of these vaccines is their lack of broad protection against the wide global diversity of varying serogroup B strains.

Hence, improved vaccines against serogroup B were pursued with the subsequent recent breakthroughs using "reverse vaccinology," which successfully created serogroup B meningococcal vaccines based on conserved proteins $[63,64]$. The advantages of these new serogroup B vaccines are several fold, including their broad coverage, which may protect even 
Table 1 Meningococcal vaccines by geographic location

\begin{tabular}{|c|c|c|c|c|}
\hline Type of vaccine & $\begin{array}{l}\text { Serogroup }(s) \\
\text { covered }\end{array}$ & Name & Licensed age group & Routine vaccination \\
\hline \multicolumn{5}{|c|}{ Sub-Saharan Africa } \\
\hline $\begin{array}{c}\text { Monovalent } \\
\text { conjugate }\end{array}$ & $\mathrm{A}$ & $\begin{array}{l}\text { PsA-TT } \\
\text { (MenAfriVac } \\
\text { Conjugate) }\end{array}$ & 3 months -29 years & $\begin{array}{l}\text { Meningitis vaccine project: } \\
\text { 1-29 years } \\
\text { Routine vaccination: } 9-18 \text { months }\end{array}$ \\
\hline \multicolumn{5}{|l|}{ Australia } \\
\hline Protein & B & $\begin{array}{l}\text { MenB-4C } \\
\text { (Bexsero) }\end{array}$ & $\begin{array}{l}\geq 2 \text { months } \\
\text { Registered for use at } \\
\geq 2 \text { months, but } 1 \text { st } \\
\text { dose can be given as } \\
\text { early as } 6 \text { weeks }\end{array}$ & $\begin{array}{l}<2 \text { years }(3 \text { doses if initiation at } \\
6 \text { weeks }-5 \text { months; } 2 \text { doses if } \\
\text { initiation at } 6-11 \text { or } \\
\geq 12 \text { months) } \\
15-19 \text { years ( } 2 \text { doses) }\end{array}$ \\
\hline \multirow[t]{4}{*}{$\begin{array}{c}\text { Monovalent } \\
\text { conjugate }\end{array}$} & $\mathrm{C}$ & $\begin{array}{l}\text { MCC-TT } \\
(\text { NeisVac-C) }\end{array}$ & $\geq 8$ weeks & 12 months \\
\hline & & $\begin{array}{l}\text { MCC-CRM } \\
\text { (Meningitec) }\end{array}$ & $\geq 6$ weeks & 12 months \\
\hline & & $\begin{array}{l}\text { MCC-CRM } \\
\text { (Menjugate) }\end{array}$ & $\geq 6$ weeks & 12 months \\
\hline & & $\begin{array}{l}\text { MCC-TT/Hib-TT } \\
\text { (Menitorix (also } \\
\text { covers H. influenzae } \\
\text { type b)) }\end{array}$ & $\geq 6$ weeks & 12 months \\
\hline \multirow[t]{2}{*}{$\begin{array}{l}\text { Quadrivalent } \\
\text { conjugate }\end{array}$} & $\mathrm{A}, \mathrm{C}, \mathrm{W}, \mathrm{Y}$ & $\begin{array}{l}\text { MenACWY-D } \\
\text { (Menactra) } \\
\text { MenACWY-CRM } \\
\text { (Menveo) }\end{array}$ & $\begin{array}{l}2-55 \text { years* } \\
11 \text { years }\end{array}$ & $\begin{array}{l}\text { Not routinely recommended. } \\
\text { Recommended in those with } \\
\text { increased risk of meningococcal } \\
\text { disease }\end{array}$ \\
\hline & & $\begin{array}{l}\text { ACWY-TT } \\
\text { (Nimenrix) }\end{array}$ & $1-55$ years* & $\begin{array}{l}\text { Not routinely recommended. } \\
\text { Recommended in those with } \\
\text { increased risk of meningococcal } \\
\text { disease }\end{array}$ \\
\hline \multirow[t]{2}{*}{$\begin{array}{l}\text { Quadrivalent } \\
\text { polysaccharide }\end{array}$} & $\mathrm{A}, \mathrm{C}, \mathrm{W}, \mathrm{Y}$ & $\begin{array}{l}\text { MPSV4 } \\
\text { (Menomune) }\end{array}$ & $\geq 2$ years & $\begin{array}{l}\text { Not routinely recommended. Can } \\
\text { be administered when repeat } \\
\text { dosing not anticipated }\end{array}$ \\
\hline & & Mencevax ACWY & $\geq 2$ years & $\begin{array}{l}\text { Not routinely recommended. Can } \\
\text { be administered when repeat } \\
\text { dosing not anticipated }\end{array}$ \\
\hline
\end{tabular}

\section{Canada}

Note: Provinces/territories may vary in schedule

See http://healthycanadians.gc.ca/apps/schedule-calendrier/index-eng.php
Protein
B
MenB-4C
2 months -17 years
Not routinely administered.
(Bexsero)
High-risk individuals 
Table 1 continued

\begin{tabular}{|c|c|c|c|c|}
\hline Type of vaccine & $\begin{array}{l}\text { Serogroup }(s) \\
\text { covered }\end{array}$ & Name & Licensed age group & Routine vaccination \\
\hline \multirow[t]{3}{*}{$\begin{array}{l}\text { Monovalent } \\
\text { conjugate }\end{array}$} & \multirow[t]{3}{*}{$\mathrm{C}$} & $\begin{array}{l}\text { MCC-TT } \\
(\text { NeisVac-C) }\end{array}$ & $\geq 2$ months & 12 months, 12 years** \\
\hline & & $\begin{array}{l}\text { MCC-CRM } \\
\text { (Meningitec) }\end{array}$ & $\geq 2$ months & 12 months, 12 years $^{* *}$ \\
\hline & & $\begin{array}{l}\text { MCC-CRM } \\
\text { (Menjugate) }\end{array}$ & $\geq 2$ months & 12 months, 12 years $^{* *}$ \\
\hline \multirow[t]{3}{*}{$\begin{array}{l}\text { Quadrivalent } \\
\text { conjugate }\end{array}$} & \multirow[t]{3}{*}{ A, C, W, Y } & $\begin{array}{l}\text { MenACWY-D } \\
\text { (Menactra) }\end{array}$ & 9 months -55 years $*$ & 12 years** $^{* *}$ \\
\hline & & $\begin{array}{l}\text { MenACWY-CRM } \\
\text { (Menveo) }\end{array}$ & 2 months -55 years* & 12 years $^{* *}$ \\
\hline & & $\begin{array}{l}\text { ACWY-TT } \\
\text { (Nimenrix) }\end{array}$ & 12 months -55 years* & 12 years $^{* *}$ \\
\hline $\begin{array}{l}\text { Quadrivalent } \\
\text { polysaccharide }\end{array}$ & $\mathrm{A}, \mathrm{C}, \mathrm{W}, \mathrm{Y}$ & $\begin{array}{l}\text { MPSV4 } \\
\text { (Menomune) }\end{array}$ & $\geq 2$ years & $\begin{array}{l}\text { Not routinely recommended. Can } \\
\text { be administered when repeat } \\
\text { dosing not anticipated }\end{array}$ \\
\hline \multicolumn{5}{|l|}{ United Kingdom } \\
\hline Protein & $\mathrm{B}$ & $\begin{array}{l}\text { MenB-4C } \\
\text { (Bexsero) }\end{array}$ & $\geq 2$ months & 2,4 and $12-13$ months \\
\hline \multirow[t]{4}{*}{$\begin{array}{l}\text { Monovalent } \\
\text { conjugate }\end{array}$} & \multirow[t]{4}{*}{$\mathrm{C}$} & $\begin{array}{l}\text { MCC-TT } \\
\text { (NeisVac-C) }\end{array}$ & $\geq 2$ months & 3 months \\
\hline & & $\begin{array}{l}\text { MCC-CRM } \\
\text { (Menjugate) }\end{array}$ & $\geq 2$ months & 3 months \\
\hline & & $\begin{array}{l}\text { MCC-CRM } \\
\text { (Meningitec) }\end{array}$ & $\geq 2$ months & $\begin{array}{l}\text { Not recommended for }<12 \text { months } \\
\text { as less immunogenic }\end{array}$ \\
\hline & & $\begin{array}{l}\text { MCC-TT/Hib-TT } \\
\text { (Menitorix (Also } \\
\text { covers } H \text {. influenzae } \\
\text { type b)) }\end{array}$ & $\geq 2$ months -2 years & $12-13$ months \\
\hline \multirow[t]{2}{*}{$\begin{array}{l}\text { Quadrivalent } \\
\text { conjugate }\end{array}$} & \multirow[t]{2}{*}{$\mathrm{A}, \mathrm{C}, \mathrm{W}, \mathrm{Y}$} & $\begin{array}{l}\text { ACWY-CRM } \\
\text { (Menveo) }\end{array}$ & $\geq 2$ years & 14 years \\
\hline & & $\begin{array}{l}\text { ACWY-TT } \\
\text { (Nimenrix) }\end{array}$ & $\geq 12$ months & 14 years \\
\hline $\begin{array}{l}\text { Quadrivalent } \\
\text { polysaccharide }\end{array}$ & A, C, W, Y & $\begin{array}{l}\text { MenACWY } \\
(\text { ACWY Vax) }\end{array}$ & $>2$ years & $\begin{array}{l}\text { Not part of routine immunization. } \\
\text { Only offered for short-term } \\
\text { travel protection in older } \\
\text { children and adults }\end{array}$ \\
\hline
\end{tabular}


Table 1 continued

\begin{tabular}{|c|c|c|c|c|}
\hline Type of vaccine & $\begin{array}{l}\text { Serogroup }(s) \\
\text { covered }\end{array}$ & Name & Licensed age group & Routine vaccination \\
\hline \multicolumn{5}{|l|}{ United States } \\
\hline \multirow[t]{2}{*}{ Protein } & \multirow[t]{2}{*}{ B } & $\begin{array}{l}\text { MenB-4C } \\
\text { (Bexsero) }\end{array}$ & $10-25$ years & $\begin{array}{l}\text { Not part of routine vaccination, } \\
\text { but may be given at } 16-18 \text { years }\end{array}$ \\
\hline & & $\begin{array}{l}\text { MenB-FHbp } \\
\text { (Trumenba) }\end{array}$ & $10-25$ years & $\begin{array}{l}\text { Not part of routine vaccination, } \\
\text { but may be given at } 16-18 \text { years }\end{array}$ \\
\hline $\begin{array}{l}\text { Bivalent } \\
\text { conjugate }\end{array}$ & $\mathrm{C}, \mathrm{Y}$ & $\begin{array}{l}\text { Hib-MenCY-TT } \\
\text { (MenHibrix (also } \\
\text { covers } H \text {. influenzae } \\
\text { type b)) }\end{array}$ & 6 weeks-18 months & Not part of routine vaccination \\
\hline \multirow[t]{2}{*}{$\begin{array}{l}\text { Quadrivalent } \\
\text { conjugate }\end{array}$} & \multirow[t]{2}{*}{$\mathrm{A}, \mathrm{C}, \mathrm{W}, \mathrm{Y}$} & $\begin{array}{l}\text { MenACWY-D } \\
\text { (Menactra) }\end{array}$ & 9 months-55 years* & $\begin{array}{l}\text { Single dose at } 11-12 \text { years, with } \\
\text { booster at } 16 \text { years }\end{array}$ \\
\hline & & $\begin{array}{l}\text { MenACWY-CRM } \\
\text { ( Menveo) }\end{array}$ & 2 months -55 years* & $\begin{array}{l}\text { Single dose at } 11-12 \text { years, with } \\
\text { booster at } 16 \text { years }\end{array}$ \\
\hline $\begin{array}{l}\text { Quadrivalent } \\
\text { polysaccharide }\end{array}$ & A, C, W, Y & $\begin{array}{l}\text { MPSV4 } \\
\text { (Menomune) }\end{array}$ & $\geq 2$ years & $\begin{array}{l}\text { Not routinely recommended. Can } \\
\text { be administered when repeat } \\
\text { dosing not anticipated }\end{array}$ \\
\hline
\end{tabular}

* Guidelines suggest these vaccines can be used among $>55$ year olds with preference for a conjugate vaccine if more than one meningococcal dose is anticipated

** Either monovalent meningococcal $\mathrm{C}$ vaccine or quadrivalent meningococcal conjugate vaccine given at 12 years (depending on local epidemiology and programmatic considerations)

against some non-serogroup B strains; of note, however, these vaccines may miss some serogroup B meningococcal strains. Recombinant protein vaccines against serogroup B include MenB-4C (Bexsero) and MenB-FHbp (Trumenba); both are approved for use in the US, with the former vaccine also approved in Europe, Canada and Australia. Of note, the OMV vaccine, MeNZB ${ }^{\circledR}$, contains P1.4, a major component of the current MenB-4C vaccine. Current serogroup B vaccines require multiple doses with MenB-4C given as a two-dose ( 0 and $\geq 1$ month) and MenB-FHbp as a three-dose (0, 2 and 6 months) initial series; the same serogroup $B$ vaccine should be utilized for all doses. Quadrivalent and serogroup B vaccines can be administered during the same visit, but should be given at different sites.

\section{Vaccine Recommendations}

Meningococcal vaccination is recommended for persons at increased risk for disease. A summary of vaccine recommendations is shown in Table 2 [65-70].

\section{WHO Guidance for High-Risk Countries}

Populations significantly affected by meningococcal disease should be prioritized for vaccination with the choice of vaccine dependent on locally prevalent $N$. meningitidis serogroups. The World Health Organization (WHO) recommends that countries with high (>10 cases per 100,000$)$ or intermediate $(2-10$ cases per 100,000) endemicity conduct large-scale meningococcal vaccine programs. Additionally, countries with frequent 
Table 2 Summary of meningococcal vaccination recommendations

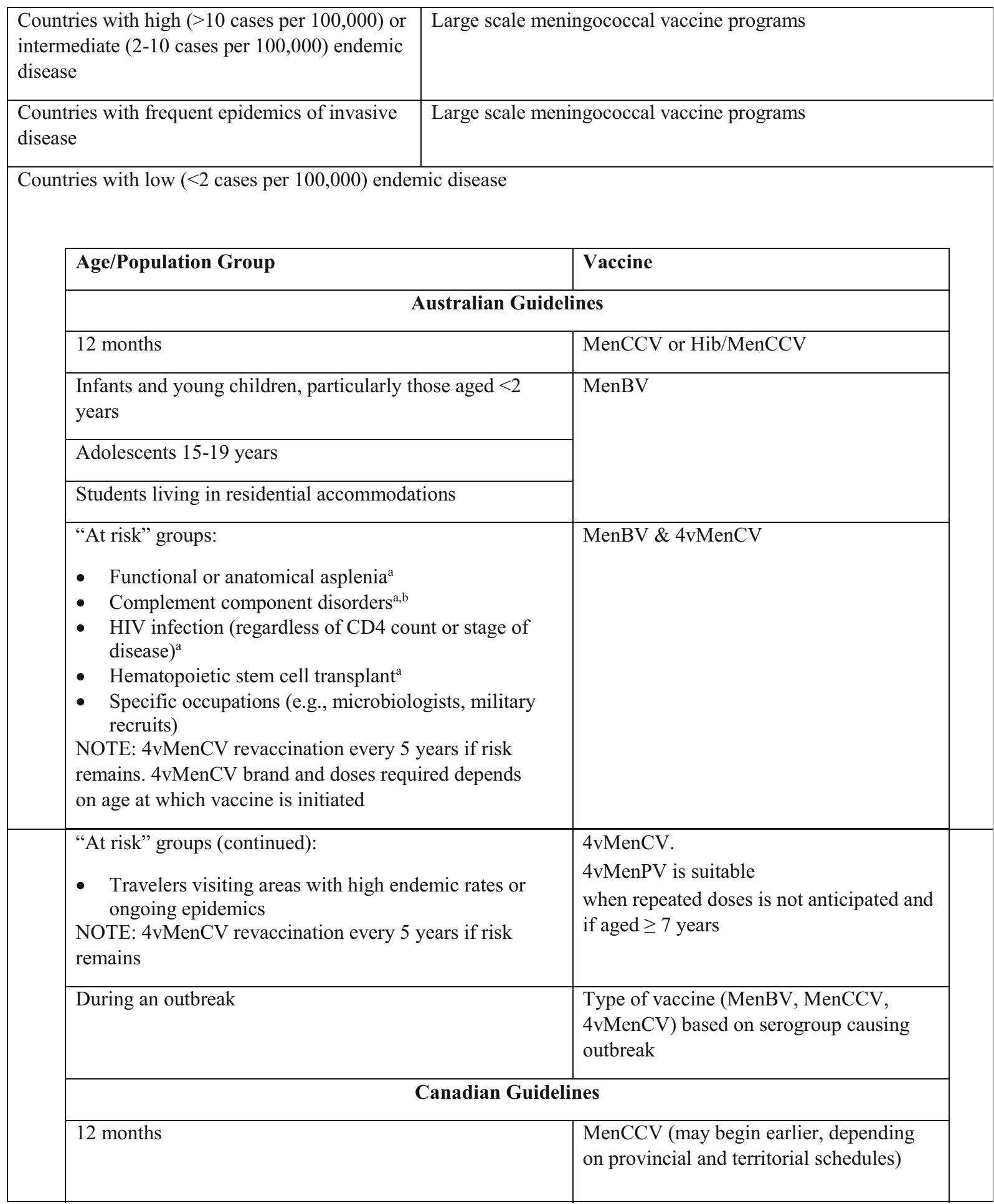


Table 2 continued

\begin{tabular}{|c|c|}
\hline 12 years & $\begin{array}{l}\text { 4vMenCV or MenCCV (depending on } \\
\text { local epidemiology and programmatic } \\
\text { considerations) and MenBV considered on } \\
\text { an individual basis, depending on } \\
\text { preferences of vaccine recipient, regional } \\
\text { serogroup B disease incidence and strain } \\
\text { susceptibility to MenBV vaccine }\end{array}$ \\
\hline $\begin{array}{l}\text { "At risk" groups: } \\
\text { - } \quad \text { Functional or anatomical asplenia }{ }^{\mathrm{a}} \\
\text { - } \quad \text { Complement component disorders }{ }^{\mathrm{a}, \mathrm{b}} \\
\text { - } \quad \text { HIV infection }{ }^{\mathrm{a}} \\
\text { - } \quad \text { Hematopoietic stem cell transplant }{ }^{\mathrm{a}} \\
\text { - } \quad \text { Specific occupations } \\
\quad \text { (e.g., microbiologists, military recruits) } \\
\text { NOTE: 4vMenCV revaccination every } 5 \text { years if risk } \\
\text { remains. } 4 \mathrm{vMenCV} \text { brand and doses required depends } \\
\text { on age at which vaccine is initiated }\end{array}$ & MenBV \& 4vMenCV \\
\hline $\begin{array}{l}\text { "At risk" groups (continued): } \\
\text { - Travelers visiting areas with high endemic rates or } \\
\text { ongoing epidemics } \\
\text { NOTE: 4vMenCV revaccination every } 5 \text { years if risk } \\
\text { remains }\end{array}$ & $\begin{array}{l}4 \mathrm{vMenCV} \text { (travelers do not need to } \\
\text { receive MenBV unless travelling to area } \\
\text { with an outbreak known to be caused by } \\
\text { meningococcal serogroup B) }\end{array}$ \\
\hline During an outbreak & $\begin{array}{l}\text { Type of vaccine (MenBV, MenCCV, } \\
4 \mathrm{vMenCV} \text { ) based on serogroup causing } \\
\text { outbreak }\end{array}$ \\
\hline \multicolumn{2}{|c|}{ United Kingdom Guidelines } \\
\hline 2 months & MenBV \\
\hline 3 months & MenCCV \\
\hline 4 months & MenBV \\
\hline 12-13 months & $\mathrm{Hib} / \mathrm{MenCCV}$ and MenBV \\
\hline 14 years & $4 \mathrm{vMenCV}$ \\
\hline $\begin{array}{l}\text { Entering university with an unknown immunization } \\
\text { history }\end{array}$ & $4 \mathrm{vMenCV}$ \\
\hline $\begin{array}{l}\text { "At risk" groups: } \\
\text { - Functional or anatomical asplenia }{ }^{\mathrm{a}} \\
\text { - Complement component disorders }{ }^{\mathrm{a}, \mathrm{b}} \\
\text { NOTE: 4vMenCV revaccination every } 5 \text { years if risk } \\
\text { remains }\end{array}$ & $\begin{array}{l}\text { MenBV, } 4 \mathrm{vMenCV}, \& \text { Hib/MenCCV } \\
4 \mathrm{vMenCV} \text { is given } 1 \text { month after } \\
\mathrm{Hib} / \mathrm{MenCV} \text { if } \geq 11 \text { years and } 2 \\
\text { months after if }<11 \text { years }\end{array}$ \\
\hline
\end{tabular}


Table 2 continued

"At risk" groups (continued)

4vMenCV and possibly MenB

- HIV infection ${ }^{\mathrm{a}}(<25$ years who have not been previously vaccinated, uncertain vaccination history, or received last MenCCV at $<10$ years).

NOTE: $4 \mathrm{vMenCV}$ revaccination every 5 years if risk remains

"At risk" groups (continued)

- Specific occupations (e.g., microbiologists) NOTE: 4vMenCV revaccination every 5 years if risk remains

"At risk" groups (continued):

4vMenCV only

- Travelers visiting areas with high endemic rates or ongoing epidemics

NOTE: 4vMenCV revaccination every 5 years if risk remains

During an outbreak

Type of vaccine: (MenBV, MenCCV, $4 \mathrm{vMenCV}$ ) based on serogroup causing outbreak

\section{United States Guidelines}

\begin{tabular}{|c|c|}
\hline 11-12 years & 4vMenCV \\
\hline 16 years & 4vMenCV \\
\hline 16-18 years & May consider MenBV \\
\hline $\begin{array}{l}\text { College students living in dormitories/residence halls who } \\
\text { have not received a dose on or after their } 16^{\text {th }} \text { birthday }\end{array}$ & 4vMenCV \\
\hline Military recruits & 4vMenCV \\
\hline $\begin{array}{l}\text { "At risk" groups: } \\
\text { - } \quad \text { Functional or anatomical asplenia }{ }^{\mathrm{a}} \\
\text { - } \quad \text { Complement component disorders }{ }^{\mathrm{a}, \mathrm{b}} \\
\text { - } \quad \text { Specific occupations (e.g., microbiologists) } \\
\text { NOTE: 4vMenCV revaccination every } 5 \text { years if risk } \\
\text { remains }\end{array}$ & MenBV \& 4vMenCV \\
\hline $\begin{array}{l}\text { "At risk" groups (continued): } \\
\text { - Travelers visiting areas with high endemic rates or } \\
\text { ongoing epidemics } \\
\text { NOTE: 4vMenCV revaccination every } 5 \text { years if risk } \\
\text { remains }\end{array}$ & 4vMenCV \\
\hline
\end{tabular}


Table 2 continued

\begin{tabular}{|l|l|l|}
\hline & During an outbreak & $\begin{array}{l}\text { Type of vaccine (MenBV, 4vMenCV) } \\
\text { based on serogroup causing outbreak }\end{array}$ \\
\cline { 2 - 3 } $\begin{array}{l}\text { Consider for emerging risk groups (e.g., HIV+ MSM } \\
\text { persons in New York State }{ }^{\mathrm{a}} \text { ): }\end{array}$ & $4 \mathrm{vMenCV}$ \\
\cline { 2 - 3 } & Other Countries: Recommendations vary by country; see specific national guidelines \\
\hline
\end{tabular}

Adapted from Specific National Guidelines [65-70]

$H i b / M e n C C V H$. influenzae $b /$ monovalent meningococcal C conjugate vaccine, $M e n B V$ meningococcal $\mathrm{B}$ vaccine, $M e n C C V$ monovalent meningococcal $\mathrm{C}$ conjugate vaccine, $4 v M e n C V$ quadrivalent meningococcal conjugate vaccine, $4 v M e n P V$ quadrivalent meningococcal polysaccharide vaccine

a 2 -dose initial vaccine series recommended

b Included deficiencies: C3, C5-9, properdin, factor D, and factor $\mathrm{H}$. In addition, the use of medications that inhibit any of these components, such as eculizumab

epidemics of invasive disease should also initiate vaccination programs. Mass vaccination campaigns should consider immunizing young persons (e.g., 9 months to 18 years of age), with a preference for the use of a conjugate vaccine given its potential for population protection [14]. Reduced carriage rates and population protection have been demonstrated after conjugate vaccine campaigns in both the UK and Africa [71-73]. Surveillance of disease rates and serogroups are advocated after such campaigns to evaluate the need for repeat vaccine campaigns and use within the routine immunization programs.

An example of mass vaccination campaigns against meningococcal disease in hyperendemic areas is the recent efforts in the African "meningitis belt" countries. Following the 1996-1997 epidemic in Africa, leaders advocated for the development and administration of a vaccine that would eliminate group A meningococcal epidemics. In response, the Meningitis Vaccine Project rolled out a MenA conjugate vaccine (MenAfriVac) in 2010 targeting ages
1-29 years. The vaccine was priced at 50 cents a dose (produced at the Serum Institute of India, Ltd.) and has the advantage of a controlled-temperature chain, allowing it to be kept in a broader range of temperatures, a key feature for its use in Africa. Thus far, $>217$ million people in 17 African countries have received the vaccine, and surveillance data have demonstrated dramatic reductions in meningococcal A epidemics and carriage rates [74]. Two formulations of MenAfriVac are available: the $10 \mu \mathrm{g}$ formulation used as a single injection for 1-29 year olds and a lower dose vaccine $(5 \mu \mathrm{g})$ approved for ages 3-24 months [73]. The WHO recommends that countries having completed mass vaccination campaigns introduce the vaccine into routine childhood immunization programs within 1-5 years (i.e., routine childhood vaccination using the $5 \mu \mathrm{g}$ dose administered as a single dose at 9 months of age). A one-time "catch-up" campaign of vaccinating birth cohorts born since the initial vaccine campaign is also advised. Recommendations for further booster doses later in life have not yet been established. 
Monitoring trends and serogroups over time is recommended to determine whether updates in the vaccine schedule or serogroup coverage may be needed.

\section{Guidelines for Lower-Risk Countries}

Meningococcal vaccination decisions in areas of low incidence $(<2$ cases per 100,000$)$ are more challenging. While low incidence rates of disease are typically seen in the developed world, invasive disease does occur and is often associated with high mortality rates and substantial disability among survivors $[15,16$, 49]. Guidelines regarding meningococcal vaccination in countries with overall low disease rates and without frequent epidemics is variable, but most (e.g., US, Europe) recommend vaccination for defined "at-risk" groups based on age, underlying host factors, and anticipated occupational and or travel-related exposures.

\section{US Guidelines}

In the US, meningococcal vaccination is guided by the Advisory Committee on Immunization Practices (ACIP) with available vaccines shown in Table 1 [49]. Since 2005, vaccination with a quadrivalent vaccine (MenACWY-D or MenACWY-CRM) has been recommended for adolescents aged 11-12 years, with revaccination at 16 years to ensure continued protection through the young adult years. Vaccination is also recommended for first-year college students living in dormitories/residence halls who have not received a dose on or after their 16th birthday (Table 2) [49]. Although infants ( $<6$ months of age) have a higher risk of infection, routine vaccination is not recommended in the US given the overall low burden of disease, poor cost-effectiveness, and since the required number of doses is not practical over this short time frame [49, 75]. Infants and children with additional risk factors for disease, as discussed below, are candidates for vaccination.

For age groups outside the adolescent/young adult years, targeted vaccination is recommended based on the presence of specific additional risk factors including functional or anatomic asplenia, persistent complement component deficiencies, and occupations associated with exposure to the organism (e.g., microbiologists, military recruits) [49]. US guidelines do not include HIV infection as an indication for vaccination [65], although specific states (e.g., New York) have encouraged its use in this population. Finally, vaccine administration should be considered in outbreak settings and directed against the causative serogroup.

Travelers visiting areas with high endemic rates or ongoing epidemics should be vaccinated with a dose received at least within the 5 years prior to travel [65]. For travelers to the African meningitis belt, the risk is highest during the dry season and with anticipated contact with the local population [14]. Vaccination is required within the last 3 years for travelers to the Hajj (given outbreaks of A, C and $\mathrm{W}$ disease), a requirement of the government of Saudi Arabia [76, 77]. Vaccines should be directed against the serogroups that are prevalent in the visited areas and should be given at least 10 days prior to departure to ensure protective antibody levels. For example, the quadrivalent conjugate vaccines $(\mathrm{A}, \mathrm{C}, \mathrm{W}$ and $\mathrm{Y}$ ) should cover the most prevalent strains currently in the African meningitis belt and Saudi Arabia. A conjugate vaccine is preferred among travelers, especially among those who take repeated trips to endemic areas. This latter recommendation is based on data suggesting that receipt of two polysaccharide vaccinations 
may result in immune hyporesponsiveness, whereas repeated conjugate vaccines may boost antibody levels [49, 78, 79].

Vaccination in the US is typically with a quadrivalent vaccine that covers serogroups $A$, C, W and Y. Both conjugate [MenACWY-D (Menactra), MenACWY-CRM (Menveo)] and polysaccharide [MPSV4 (Menomune)] quadrivalent vaccines are available and licensed for specific age groups (Table 1) [49]. A bivalent conjugate meningococcal vaccine is available in the US [HibMenCY-TT (MenHibrix)] and also offers coverage against Haemophilus influenzae $b$, but only contains two meningococcal serogroups $(\mathrm{C}, \mathrm{Y})$. In general, conjugate vaccines are preferred; however, polysaccharide vaccines can be used when the conjugate vaccine is unavailable and for those $\geq 56$ years who require only a single vaccine dose [65].

Since serogroup B now accounts for $40 \%$ of the cases in the US [47], vaccination with MenB-4C or MenB-FHbp has been added to the immunization schedule for those at highest risk including persons with asplenia, persistent complement component deficiencies, and occupations such as microbiologists [64]. Serogroup B vaccination can also be considered among other at-risk groups (e.g., adolescents, college students); however, it is not currently uniformly recommended given the overall low burden of disease. For example, if the vaccine were administered in the US at ages 11 and 16-18 years, an estimated 15-29 cases and 2-5 deaths might be prevented each year; if given only to college students, an estimated nine cases and one death might be averted each year [80]. If the serogroup B vaccine is utilized, the preferred strategy per ACIP guidelines is to administer it later in adolescence (i.e., aged 16-18 years) to maximize protection during the highest age risk period [80]. Overall, individual clinical decision making has been advised regarding the use of the serogroup B vaccine among US adolescents and young adults who do not have additional risk factors. Additionally, in the setting of increased serogroup B cases or outbreaks (defined as $\geq 2$ primary cases in organizations with $<5000$ persons over a 6 month period), vaccination is advised [64, 65, 81].

\section{European Guidelines}

The use of meningococcal vaccination in Europe differs from the US given the geographically varying epidemiologic trends. In 1999, the MenC conjugate vaccine was added to the UK's routine immunization schedule, resulting in significant and sustained reductions in serogroup $C$ cases $[22,68]$. In 2013, the serogroup B vaccine, MenB-4C, was approved for persons $\geq 2$ months of age and subsequently added to the vaccine schedule [82]. Current UK guidelines suggest the administration of two doses of serogroup B at 2 and 4 months followed by a booster at 12-13 months of age and one dose of serogroup $\mathrm{C}$ meningococcal conjugate vaccine at 3 months of age [MCC-TT (Neisvac-C) or MCC-CRM (Menjugate)], followed by an MCC-TT/Hib-TT (MenC/H. influenzae type b conjugate) vaccine at $12-13$ months [68, 83-85].

The recent expansion of the hyper-virulent serogroup W strain (cc11) led to an adolescent MenACWY conjugate vaccination program initiated in 2015 for those 14 years of age [86]. Hence, a quadrivalent meningococcal conjugate (ACWY-CRM or ACWY-TT) is administered among these age groups. For those entering university with an unknown immunization history, a conjugate 
quadrivalent ACWY vaccine dose is also recommended (Table 2 ).

In addition, those at risk for disease (e.g., asplenia, complement disorders) should receive vaccination based on the age at which their risk condition is diagnosed [68]. The UK guidelines also recommend a dose of conjugate MenACWY for those traveling to hyperendemic or epidemic areas, even if previously vaccinated with MenC [68]. Finally, the British HIV Association suggests vaccination among HIV-infected persons aged $<25$ years [69].

Other European countries have also introduced meningococcal $\mathrm{C}$ vaccinations into their immunization programs, some as part of the infant immunization schedule (e.g., Greece, Iceland, Ireland, Portugal, Spain and the UK), while others advise initiation during the 2nd year of life (e.g., Belgium, Cyprus, Italy and The Netherlands) [22, 85]. The European Commission approved MenB-4C in 2013, and the UK subsequently was the first country to introduce MenB-4C into their national infant immunization program (2015). As noted above, most countries also recommend vaccination among those at high risk for disease (e.g., asplenia, terminal complement deficiencies) and travelers to endemic areas with some variations by country. A summary of European recommendations by country can be found on the European Centre for Disease Prevention and Control website [85].

\section{Guidelines for Other Locations}

Country-specific guidelines have been developed for other regions of the world [22, 87]. Guidance for Australia and Canada are shown in Table 2 [66, 70]. Overall, vaccine strategies against meningococcal disease vary worldwide and should be based on local epidemiologic trends derived from ongoing surveillance data. Since Neisseria are capable of undergoing capsular switching (e.g., ST11, which is typically associated with serogroup C, can alter its capsule to serogroup B) [88] and serogroup/ST replacement can occur over time, continued reassessment of trends and serogroups is advocated to ensure optimal vaccine programs. The importance of surveillance in serogroup trends is exemplified by the global spread of serogroup W (cc11) causing severe cases of invasive disease [29, 31].

\section{Number of Vaccinations}

Establishment of protection against invasive disease is via the development of serum bactericidal antibodies against $N$. meningitidis capsular polysaccharides or protein antigens. Due to the low incidence of meningococcal disease in much of the world, vaccine effectiveness has largely been based on immunogenicity data as measured by serum bactericidal activity (SBA) [14].

The number of vaccines utilized in the initial series is based on host characteristics including age and underlying medical conditions. In most adults and children (age $\geq 24$ months), a single dose is recommended for initial vaccination. Additional doses are generally recommended among infants and toddlers $\leq 23$ months [68, 89]. Furthermore, among children and adults with underlying medical conditions [e.g., asplenia, persistent complement component deficiencies and immunosuppression (e.g., HIV)], significantly lower antibody titers have been achieved after a single dose [90, 91]. Hence, when these groups are vaccinated, additional vaccine doses are often recommended [65-70]. For example, in the US, these groups receive a two-dose initial series administered 8-12 weeks apart $[49,65]$. 
Current vaccines do not provide lifelong protection even though the risk for meningococcal disease persists among all ages. In general, polysaccharide vaccines induce 3-5 years of protection among adults. Although it was hoped that conjugate vaccines would induce immune responses with longer durations of protection, this may not be the case after single dose administration [47]. For example, among adolescents, one study demonstrated vaccine effectiveness for MenACWY at 1 year post-vaccination of $82 \%$, but found waning protection (50-60\%) at $2-5$ years [49]. The durability of protection of the broad-spectrum, protein serogroup B vaccines (MenB-4C and MenB-FHbp) remains unknown. Therefore, some guidelines recommend booster doses among those who remain at high risk for the disease. For example, in countries such as Australia, Canada, the UK and US, a booster dose of conjugate quadrivalent ACWY vaccine is recommended every 5 years for persons at continued high risk such as microbiologists, patients with asplenia or persistent complement component deficiencies, immunosuppressed (HIV) patients, and frequent travelers to endemic areas [65-70]. Among children at higher risk for disease, revaccination is recommended 3 years after the initial dose if received at an age $\leq 6$ years $[49,92]$.

\section{Vaccine Administration}

Most polysaccharide vaccines are administered by subcutaneous injection, whereas conjugate vaccines are given by intramuscular injection, typically in the deltoid muscle, except among those $<12$ months, in whom the anterolateral thigh is preferred [14].

Co-administration of the meningococcal vaccine with other routinely administered vaccines appears to be generally safe and without significant effect on vaccine efficacy; administration should use separate injection sites [65, 74, 83, 84, 93]. For example, studies of MenAfriVac suggest that it can be administered at the same visit as routine vaccines (e.g., diphtheria, tetanus toxoid, whole cell pertussis, hepatitis B, measles, rubella, oral poliovirus, $H$. influenzae $b$ and yellow fever); the conjugate pneumococcal vaccine (Prevnar) and rotavirus vaccines were not evaluated [74]. Studies on the conjugate quadrivalent meningococcal vaccine (MenACWY-D, Menactra) with PCV13 (Prevnar) did find reduced antibody titers to some pneumococcal serogroups, but levels were still in the protective range [94]. Due to the high risk of invasive pneumococcal disease in children with functional or anatomic asplenia, the Centers for Disease Control and Prevention (CDC) in the US recommend considering administration of MenACWY-D at least 4 weeks after completion of all PCV13 doses [95]. Finally, although some meningococcal conjugate vaccines (i.e., MenACWY-D and MenACWY-CRM) contain diphtheria toxoid, no adverse findings have been noted with concurrent administration of the tetanus-diphtheria-pertussis (Tdap) vaccine [96].

\section{Adverse Events}

Millions of doses of polysaccharide and, more recently, conjugate vaccines have been administered worldwide, demonstrating an excellent safety profile to date. Adverse reactions when they do occur are typically mild and most commonly are injection site erythema or tenderness [97]. Post-vaccination headache, fever or dizziness may occur. Syncope has been reported especially among adolescents. While Guillian-Barre syndrome 
has been reported, the risk does not appear to be elevated based on US data evaluating the meningococcal conjugate MenACWY-D (Menactra) vaccine or Canadian data on MCC-CRM (Menjugate) [98, 99]. Allergic reactions and/or anaphylaxis are uncommon (e.g., estimated as $<0.1 / 100,000$ vaccines doses for polysaccharide vaccines) [14].

\section{Use in Pregnancy}

Although meningococcal vaccines have not been extensively studied in pregnancy or lactating women and no randomized trials are available, an observational study of the MenAfriVac did not show any adverse signals for pregnancy outcomes. As such, pregnant women in the targeted age range are being included in the mass vaccine campaigns in Africa [74]. Similarly, the Vaccine Adverse Event Reporting System (VAERS) system in the US has identified no major safety concerns in the mother or fetus $[49,100]$. Hence, based on the available data, pregnancy or lactation is not considered as an exclusion for vaccination [74, 101].

\section{OTHER PREVENTION STRATEGIES INCLUDING ANTIBIOTIC PROPHYLAXIS}

Close contacts of patients with invasive disease have a markedly increased risk for developing meningococcal infection (estimated as $>100$-fold increase compared with the general population) [49, 102]. As such, prophylactic antibiotics are recommended for close contacts including household members or persons in the same daycare, college dormitory or military unit $[49,92]$. Additionally, those directly exposed to a patient's oral secretions (kissing, sharing food or drink utensils, or mouth-to-mouth resuscitation) within the 7 days before symptom onset should be offered prophylaxis [49].

Secondary cases most often occur within $72 \mathrm{~h}$ of presentation of the index cases; hence, antibiotic prophylaxis should be initiated immediately, ideally within $24 \mathrm{~h}$ of the identification of the index case. Presentation may occur up to 14 days of exposure; hence, prophylaxis can be offered up to this time point [49]. Chemoprophylaxis in the setting of ongoing epidemics and exposures (e.g., the African meningitis belt) are often not pursued given the multiple sources and prolonged risk of exposures; vaccine protection is the best preventive strategy in these settings.

Prophylactic antibiotic regimens may include oral ciprofloxacin (adult dose: $500 \mathrm{mg} \times 1$ dose), ceftriaxone (adult dose: $250 \mathrm{mg}$ IM $\times 1$; children $<15$ years: $125 \mathrm{mg}$ $\mathrm{IM} \times 1$ ), rifampin (dose dependent on age; adult dose: $600 \mathrm{mg}$ po bid $\times 2$ days) or azithromycin $(10 \mathrm{mg} / \mathrm{kg}$ with maximum dose of $500 \mathrm{mg} \times 1)$ $[49,103]$. Effectiveness is estimated as $90-95 \%$ [104]. The preferred chemoprophylaxis depends on existing resistance patterns among strains in the geographic area of acquisition as well as host characteristics such as age, underlying medical conditions, pregnancy status and concurrent medications. For example, reports of rifampin- and ciprofloxacin-resistant $N$. meningitidis isolates have been described [105, 106].

Other methods for preventing disease include prompt diagnosis and management of patients with invasive disease. Historically, treatment was with immune antiserum and then with sulfonamides during the early antibiotic era [1, 107]. As sulfonamides are no longer viable options due to resistance, treatment today is typically with the 
immediate institution of intravenous ceftriaxone or cefotaxime, with alternate options including chloramphenicol or meropenem [108]. Penicillin may be utilized if the isolate is shown to be susceptible by microbiologic testing; confirmation is advised since $3-16 \%$ of isolates have MICs in the intermediate susceptibility range $[1,105]$. Infected patients should be maintained in droplet precautions until at least $24 \mathrm{~h}$ after treatment to prevent spread. The index case should also receive therapy to eradicate the carrier state if penicillin, ampicillin or chloramphenicol was used because these antibiotics do not reliably clear carriage; the same regimens for eradicating carriage can be used as for close contacts. Finally, reduction in crowding and other risk factors discussed above are also important for disease reduction.

\section{CONCLUSION}

N. meningitidis remains a continually evolving disease with diverse and varying geographic trends. Vaccines are currently available for five of the six serogroups that cause the majority of disease, and many countries have implemented meningococcal vaccine programs with subsequent reductions in the burden of disease. Recent accomplishments include the widespread availability of meningococcal conjugate vaccines that cover four of the most common serogroups (A, C, W, Y) causing human disease, are immunogenic in infants/ children and provide population protection; the long awaited licensure of vaccines to combat serogroup B; and the successful mass vaccination campaigns including in areas such as the African meningitis belt. Future efforts should focus on establishing robust worldwide surveillance systems to track incidence rates and disease-related ST complexes/serogroups in order to inform vaccine guidelines. In addition, the continued worldwide implementation of vaccine initiatives to control disease is advocated. Further, enhanced surveillance preand post- introduction of vaccine interventions, especially the complex broad-spectrum anti-serogroup B vaccines, is essential for the development of future control strategies against meningococcal disease. Finally, the development of novel vaccines with broad serogroup coverage and long durability should be pursued.

\section{ACKNOWLEDGMENTS}

No funding or sponsorship was received for this study or publication of this article. All name authors meet the International Committee of Medical Journal Editors (ICMJE) criteria for authorship for this manuscript, take responsibility for the integrity of the work as a whole and have given final approval for the version to be published.

Disclosures. Nancy Crum-Cianflone and Eva Sullivan have nothing to disclose.

Compliance with Ethics Guidelines. This article is based on previously conducted studies and does not involve any new studies of human or animal subjects performed by any of the authors.

Open Access. This article is distributed under the terms of the Creative Commons Attribution-NonCommercial 4.0 International License (http://creativecommons.org/licenses/ by-nc/4.0/), which permits any noncommercial use, distribution, and reproduction in any medium, provided you give appropriate credit 
to the original author(s) and the source, provide a link to the Creative Commons license, and indicate if changes were made.

\section{REFERENCES}

1. Stephens DS, Apicella MA. Neisseria meningitidis. In: Mandell, Douglas, and Bennett's principles and practice of infectious diseases. 8th ed. Philadelphia, PA Elsevier Saunders Inc.; 2015. p. 2425-45.

2. Marchiafava E, Celli A. Spra i micrococchi della meningite cerebrospinale epidemica. Gazz degli Ospedali. 1884;5:59.

3. Weichselbaum A. Ueber die aetiologie der akuten meningitis cerebrospinalis. Fortschr Med. $1887 ; 5: 573$.

4. Christensen H, May M, Bowen $\mathrm{L}$, et al. Meningococcal carriage by age: a systematic review and meta-analysis. Lancet Infect Dis. 2010;10:853-61.

5. Goldschneider I, Gotschlich EC, Artenstin MS. Human immunity to the meningococcus. II. Development of natural immunity. J Exp Med. 1969;129:1327-48.

6. Goldschneider I, Gotschlich EC, Artenstein MS. Human immunity to the meningococcus, I. The role of humoral antibodies. J Exp Med. 1969;129:1307-26.

7. Stephens DS. Uncloaking the meningococcus, the dynamics of meningococcal carriage and meningococcal disease. Lancet. 1999;353:941-2.

8. Rosenstein NE, Perkins BA, Stephens DS, Popovic T, Hughes JM. Meningococcal disease. N Engl J Med. 2001;344:1378-88.

9. Pace D, Pollard AJ. Meningococcal disease: clinical presentation and sequelae. Vaccine. 2012;30(Suppl 2):B3-9.

10. Girard MP, Preziosi MP, Aguado MT, Kieny MP. A review of vaccine research and development: meningococcal 2006;24:4692-700.

11. Thigpen MC, Whitney CG, Messonnier NE, et al. Emerging infections programs network. bacterial meningitis in the United States, 1998-2007. N Engl J Med. 2011;364:2016-25.
12. Brouwer MC, Tunkel AR, van de Beek D. Epidemiology, diagnosis, and antimicrobial treatment of acute bacterial meningitis. Clin Microbiol Rev. 2010;23:467-92.

13. WHO Initiative for Vaccine Research (IVR): Meningococcal disease. Available at http://www. who.int/vaccine_research/diseases/soa_bacterial/ en/index1.html. Accessed 1 Feb 2016.

14. Meningococcal vaccines: WHO position paper, November 2011. Wkly Epidemiol Rec. 2011;86:521-39.

15. Erickson LJ, De Wals P, McMahon J, Heim S. Complications of meningococcal disease in college students. Clin Infect Dis. 2001;33:737-9.

16. Edwards MS, Baker CJ. Complications and sequelae of meningococcal infections in children. J Pediatr. 1981;99:540-5.

17. Gianchecchi E, Torelli A, Piccini G, Piccirella S, Montomoli E. Neisseria meningitidis infection: who, when and where? Expert Rev Anti Infect Ther. 2015;13:1249-63.

18. Ashton FE, Ryan A, Diena B, et al. A new serogroup (L) of Neisseria meningitidis. J Clin Microbiol. 1983;17:722-7.

19. Ding S, Ye R, Zhang H. Three new serogroups of Neisseria meningitidis. J Biol Stand. 1981;9:305-15.

20. Abio A, Neal KR, Beck CR. An epidemiological review of changes in meningococcal biology during the last 100 years. Pathog Glob Health. 2013;107:373-80.

21. Trotter CL, Ramsay ME. Vaccination against meningococcal disease in Europe: review and recommendations for the use of conjugate vaccines. FEMS Microbiol Rev. 2007;31:101-7.

22. Halperin SA, Bettinger JA, Greenwood B, et al. The changing and dynamic epidemiology of meningococcal disease. Vaccine. 2012;30(Suppl 2):B26-36.

23. Harrison LH, Trotter CL, Ramsay ME. Global epidemiology of meningococcal disease. Vaccine. 2009;27(Suppl 2):B51-63.

24. European Centre for Disease Prevention and Control. Surveillance of Invasive bacterial diseases in Europe 2008/2009. Stockholm: ECDC; 2011.

25. Ladhani SN, Beebeejaun $\mathrm{K}$, Lucidarme J, et al. Increase in endemic Neisseria meningitidis 
capsular group W sequence type 11 complex associated with severe invasive disease in England and Wales. Clin Infect Dis. 2015;60:578-85.

26. Ceyan M, Anis S, Htun-Myint $L$, et al. Meningococcal disease in the Middle East and North Africa: an important public health consideration. Int J Infect Dis. 2012;16:e574-82.

27. Sáfadi MA, González-Ayala $S$, Jäkel A, et al. The epidemiology of meningococcal disease in Latin America, 1945-2010: an unpredictable and changing landscape. Epidemiol Infect. 2012;141:447-58.

28. Vyse A, Wolter JM, Chen J, et al. Meningococcal disease in Asia: an under-recognised public health burden. Epidemiol Infect. 2011;139:967-85.

29. Lucidarme J, Hill DM, Bratcher HB, et al. Genomic resolution of an aggressive, widespread, diverse and expanding meningococcal serogroup B, C and W lineage. J Infect. 2015;71:544-52.

30. Mustapha MM, Marsh JW, Harrison LH. Global epidemiology of capsular group $\mathrm{W}$ meningococcal disease (1970-2015): multifocal emergence and persistence of hypervirulent sequence type (ST)-11 clonal complex. Vaccine. 2016;34:1515-23.

31. Zhou H, Liu W, Xu L, et al. Spread of Neisseria meningitidis serogroup W clone, China. Emerg Infect Dis. 2013;19:1496-9.

32. Mandal S, Wu HM, MacNeil JR, et al. Prolonged university outbreak of meningococcal disease associated with a serogroup B strain rarely seen in the United States. Clin Infect Dis. 2013;57:344-8.

33. Soeters HM, McNamara LA, Whaley $M$, et al. Serogroup B meningococcal disease outbreak and carriage evaluation at a college-Rhode Island, 2015. MMWR. 2015;64:606-7.

34. Cohn A, MacNeil J. The changing epidemiology of meningococcal disease. Infect Dis Clin N Am. 2015;29:667-77.

35. Centers for Disease Control and Prevention. Meningococcal Disease: Technical \& Clinical Information. http://www.cdc.gov/meningococcal/ clinical-info.html. Accessed 20 Feb 2016.

36. Stephens DS. Conquering the meningococcus. FEMS Microbiol Rev. 2007;31:3-14.

37. Mutonga DM, Pimentel G, Muindi J, et al. Epidemiology and risk factors for serogroup $\mathrm{X}$ meningococcal meningitis during an outbreak in western Kenya, 2005-2006. Am J Trop Med Hyg. 2009;80:619-24.

38. Ram S, Lewis LA, Rice PA. Infections of people with complement deficiencies and patients who have undergone splenectomy. Clin Microbiol Rev. 2010;23:740-80.

39. Miller EC, Chase NM, Densen $P$, et al. Autoantibody stabilization of the classical pathway C3 convertase leading to C3 deficiency and neisserial sepsis: c4 nephritic factor revisited. Clin Immunol. 2012;145:241-50.

40. Struijk GH, Bouts AHM, Rijkers GT, et al. Meningococcal sepsis complicating eculizumab treatment despite prior vaccination. Am J Transplant. 2013;13:819-20.

41. Salit IE. Meningococcemia caused by serogroup W-135: association with hypogammaglobulinemia. Arch Intern Med. 1981;141:664-5.

42. Miller L, Arakaki L, Ramautar A, et al. Elevated risk for invasive meningococcal disease among persons with HIV. Ann Intern Med. 2014;160:30-7.

43. Simon MS, Weiss D, Gulick RM. Invasive meningococcal disease in men who have sex with men. Ann Intern Med. 2013;159(4):300-1.

44. Emonts M, Hazelzet JA, de Groot R, Hermans PW. Host genetic determinants of Neisseria meningitidis infections. Lancet Infect Dis. 2003;3:565-77.

45. Hibberd ML, Sumiya M, Summerfield JA, et al. Association of variants of the gene for mannose-binding lectin with susceptibility to meningococcal disease. Lancet. 1999;353: 1049-53.

46. Fijen CA, Bredius RG, Kuijper EJ, et al. The role of Fc gamma receptor polymorphisms and C3 in the immune defense against Neisseria meningitidis in complement-deficient individuals. Clin Exp Immunol. 2000;120:338-45.

47. Cohn AC, MacNeil JR, Harrison LH, et al. Changes in Neisseria meningitidis disease epidemiology in the United States, 1998-2007: implications for prevention of meningococcal disease. Clin Infect Dis. 2010;50:184-91.

48. Bruce MG, Rosenstein NE, Capparella JM, Shutt KA, Perkins BA, Collins M. Risk factors for meningococcal disease in college students. JAMA. 2001;286:688-93.

49. Cohn AC, MacNeil JR, Clark TA, et al; Centers for Disease Control and Prevention (CDC). Prevention 
and control of meningococcal disease: recommendations of the Advisory Committee on Immunization Practices (ACIP). MMWR Recomm Rep. 2013;62(RR-2):1-28.

50. Sejvar JJ, Johnson D, Popovic T, et al. Assessing the risk of laboratory-acquired meningococcal disease. J Clin Microbiol. 2005;43:4811-4.

51. Aguilera J-F, Perrocheau A, Meffre C. Hahné S; W135 Working Group. Outbreak of the serogroup W135 meningococcal disease after the Hajj pilgrimage, Europe, 2000. Emerg Infect Dis. 2002;8:761-7.

52. Teyssou R, Muros-Le RE. Meningitis epidemics in Africa: a brief overview. Vaccine. 2007;25:A3-7.

53. Moore PS, Hierholzer J, DeWitt W, Gouan K, et al. Respiratory viruses and mycoplasma as cofactors for epidemic group A meningoccal meningitis. JAMA. 1990;264:1271-5.

54. Fischer M, Hedberg K, Cardosi P, et al. Tobacco smoke as a risk factor for meningococcal disease. Pediatr Infect Dis J. 1997;16:979-83.

55. Molesworth AM, Cuevas LE, Connor SJ, et al. Environmental risk and meningitis epidemics in Africa. Emerge Infect Dis. 2003;9:1287-93.

56. Greenwood BM, Bradley AK, Wall RA. Meningococcal disease and season in sub-Saharan Africa. Lancet. 1985;2:829-30.

57. Artenstein MS, Gold R, Zimmerly JG, et al. Prevention of meningococcal disease by group $\mathrm{C}$ polysaccharide vaccine. $\mathrm{N}$ Engl $\mathrm{J}$ Med. 1970;282:417-20.

58. Vipond C, Care R, Feavers IM. History of meningococcal vaccines and their serological correlates of protection. Vaccine. 2012;30:B10-7.

59. Borrow R. Advances with vaccination against Neisseria meningitidis. Trop Med Int Health. 2012;17:1478-91.

60. Micoli F, Romano MR, Tontini $M$, et al. Development of a glycoconjugate vaccine to prevent meningitis in Africa caused by meningococcal serogroup X. Proc Natl Acad Sci USA. 2013;1110:19077-82.

61. Granoff DM. Review of meningococcal group B vaccines. Clin Infect Dis. 2010;50:S54-65.

62. Holst J, Oster P, Arnold R, et al. Vaccines against meningococcal serogroup $\mathrm{B}$ disease containing outer membrane vesicles (OMV): lessons from past programs and implications for the future. Hum Vaccin Immunother. 2013;9:1241-53.
63. Caron F, du Châtelet IP, Leroy JP, et al. From tailor-made to ready-to-wear meningococcal $\mathrm{B}$ vaccines: longitudinal study of a clonal meningococcal B outbreak. Lancet Infect Dis. 2011;11:455-63.

64. Folaranmi T, Rubin L, Martin SW, Patel M, MacNeil JR, Centers for Disease Control (CDC). Use of serogroup $B$ meningococcal vaccines in persons aged $\geq 10$ years at increased risk for serogroup $\bar{B}$ meningococcal disease: recommendations of the advisory committee on immunization practices, 2015. MMWR Morb Mortal Wkly Rep. 2015;64:608-12.

65. Kim DK, Bridges CB, Harriman $\mathrm{KH}$, on behalf of the Advisory Committee on Immunization Practices. Advisory committee on immunization practices recommended immunization schedule for adults aged 19 years or older: United States, 2016. Ann Intern Med. 2016;164:184-94.

66. Australian Immunisation Handbook. Australian Government Department of Health. 4.10 Meningococcal disease. Available at http://www. immunise.health.gov.au/internet/immunise/pub lishing.nsf/Content/Handbook10-home handbook 10part4 handbook10-4-10\#list4-10-1. Accessed 21 Feb 2016.

67. Joint Committee on Vaccination and Immunisation. Available at https:/www.gov.uk/ government/groups/joint-committee-on-vaccinati on-and-immunisation. Accessed 21 Feb 2016.

68. Green Book: Chapter 7 Immunization of Individuals with Underlying Medical Conditions; p. 49-56. Available at https://www.gov.uk/ government/uploads/system/uploads/attachment data/file/309218/Green_Book_Chapter_7_v1_3.pdf. Chapter 22 Meningococcal; p. 1-29. Available at https://www.gov.uk/government/uploads/system/ uploads/attachment_data/file/462629/2904512 Green_Book_Chapter_22_v6_0W.PDF. Chapter 12 Immunisation of healthcare and laboratory staff; p. 83-90. Available at https://www.gov.uk/ government/uploads/system/uploads/attachment_ data/file/147882/Green-Book-Chapter-12.pdf.

69. Geretti AM; BHIVA Immunization Writing Committee. British HIV Association guidelines for immunization of HIV-infected adults 2015. British HIV Association 2015. Available at http:// www.bhiva.org/documents/Guidelines/Vaccination/ 2015-Vaccination-Guidelines.pdf.

70. Public Agency of Canada-Canada Immunization Guide. Available at www.publichealth.gc.ca. Accessed 21 Feb 2016.

71. Maiden $\mathrm{MC}$, Ibarza-Pavon $\mathrm{AB}$, Urwin $\mathrm{R}$, et al. Impact of meningococcal serogroup $C$ conjugate 
vaccines on carriage and herd immunity. J Infect Dis. 2008;197:737-43.

72. Daugla DM, Gami JP, Gamougam K, et al. Effect of a serogroup A meningococcal conjugate vaccine (PsA-TT) on serogroup A meningococcal meningitis and carriage in Chad: a community study. Lancet. 2014;383:40-7.

73. Okwo-Bele J-M, LaForce FM, Borrow R, Preziosi M-P. Documenting the results of a successful partnership: a new meningococcal vaccine for Africa. Clin Infect Dis. 2015;61:S389-90.

74. WHO Weekly epidemiological record-Meningococcal A conjugate vaccine: updated guidance, February 2015;90, 57-68. Available at http://www.who.int/wer/2015/ wer9008.pdf?ua=1.

75. Centers for Disease Control and Prevention. Infant meningococcal vaccination: advisory Committee on Immunization Practices (ACIP) recommendations and rationale. MMWR Morb Mortal Wkly Rep. 2013;62:52-4.

76. Al-Tawfiq JA, Memish ZA. The Hajj: updated health hazards and current recommendations for 2012. Euro Surveill. 2012;17. pii: 20295.

77. Cramer JP, Wilder-Smith A. Meningococcal disease in travelers: update on vaccine options. Curr Opin Infect Dis. 2012;25:507-17.

78. Borrow R, Joseph H, Andrews N, et al. Reduced antibody response to revaccination with meningococcal serogroup A polysaccharide vaccine in adults. Vaccine. 2000;19:1129-32.

79. Richmond P, Kaczmarski E, Borrow R, et al. Meningococcal $\mathrm{C}$ polysaccharide vaccine induces immunologic hyporesponsiveness in adults that is overcome by meningococcal $\mathrm{C}$ conjugate vaccine. J Infect Dis. 2000;181:761-4.

80. MacNeil JR, Rubin L, Folaranmi T, Ortega-Sanchez IR, Patel M, Martin SW. Use of Serogroup B meningococcal vaccines in adolescents and young adults: recommendations of the Advisory Committee on Immunization Practices, 2015. MMWR Morb Mortal Wkly Rep. 2015;64:1171-6.

81. McNamara LA, Shumate AM, Johnsen P, et al. Use of a serogroup B meningococcal vaccine in the US in response to a university outbreak. Pediatrics. $2015 ; 135: 798-804$.

82. Pollard AJ, Riordan A, Ramsay M. Group B meningococcal vaccine: recommendations for UK use. Lancet. 2014;383:1103-4.
83. Gossger N, Snape MD, Yu LM, et al. European MenB Vaccine Study Group. Immunogenicity and tolerability of recombinant serogroup B meningococcal vaccine administered with or without routine infant vaccinations according to different immunization schedules: a randomized controlled trial. JAMA. 2012;307:573-82.

84. Vesikari T, Esposito S, Prymula R, et al. EU Meningococcal B Infant Vaccine Study group. Immunogenicity and safety of an investigational multicomponent, recombinant, meningococcal serogroup $B$ vaccine (4CMenB) administered concomitantly with routine infant and child vaccinations: results of two randomised trials. Lancet. 2013;381:825-35.

85. Vaccine Schedule. The European Centre for Disease Prevention and Control. Available at: http://vaccine-schedule.ecdc.europa.eu/Pages/ Scheduler.aspx. Accessed on 4 Jan 2016.

86. Campbell H, Saliba V, Borrow R, Ramsay M, Ladhani SN. Targeted vaccination of teenagers following continued rapid endemic expansion of a single meningococcal group $\mathrm{W}$ clone (sequence type 11 clonal complex), United Kingdom 2015. Euro Surveill. 2015;20. pii: 21188.

87. Safadi MA, Bettinger JA, Moreno Meturana G, et al. Evolving meningococcal immunization strategies. Expert Rev Vaccines. 2015;14:505-17.

88. Harrison LH, Shutt KA, Schmink SE, et al. Population structure and capsular switching of invasive Neisseria meningitidis isolates in the pre-meningococcal conjugate vaccine eraUnited States, 2000-2005. J Infect Dis. 2010;201:1208-24.

89. Academy of Pediatrics Committee on Infectious Diseases. Updated recommendations on the use of meningococcal vaccines. Pediatrics. 2014;134:400.

90. Balmer P, Falconer M, McDonald P, et al. Immune response to meningococcal serogroup $\mathrm{C}$ conjugate vaccine in asplenic individuals. Infect Immun. 2004;72:332-7.

91. Lujan-Zilbermann J, Warshaw MG, Williams PL, International Maternal Pediatric Adolescent AIDS Clinical Trials Group P1065 Protocol Team, et al. Immunogenicity and safety of 1 vs 2 doses of quadrivalent meningococcal conjugate vaccine in youth infected with human immunodeficiency virus. J Pediatr. 2012;161:676-81.

92. American Academy of Pediatrics. [Meningococcal Infections.] In: Kimberlin DW, Brady MT, Jackson MA, Long SS, eds. Red Book: 2015 Report of the 
Committee on Infectious Diseases. 30th ed. Elk Grove Village: American Academy of Pediatrics; 2015. p. 547-57.

93. Gasparini R, Tregnaghi M, Keshavan P, Ypma E, Han L, Smolenov I. Safety and immunogenicity of a quadrivalent meningococcal conjugate vaccine and commonly administered vaccines after coadministration. Pediatr Infect Dis J. 2016;35:81-93.

94. Food and Drug Administration. Product approval information: package insert. Menactra (meningococcal (groups A, C, Y and W-135) polysaccharide diphtheria toxoid conjugate vaccine). Sanofi Pasteur. Silver Spring, MD: US Department of Health and Human Services, Food and Drug Administration; 2011. Available at http://www.fda.gov/downloads/biologicsbloodvacci nes/vaccines/approvedproducts/ucm131170.pdf. Accessed 21 Feb 2016.

95. Centers for Disease Control and Prevention: Recommended Immunization Schedules for Persons Aged 0 Through 18 years; United States 2016. Available at: http://www.cdc.gov/vaccines/ schedules/downloads/child/0-18yrs-child-combinedschedule.pdf. Accessed 22 Feb 2016.

96. Weston WM, Friedland LR, Wu X, Howe B. Immunogenicity and reactogenicity of co-administered tetanus-diphtheria-acellular pertussis (Tdap) and tetravalent meningococcal conjugate (MCV4) vaccines compared to their separate administration. Vaccine. 2011;29:1017-22.

97. Zahlanie YC, Hammadi MM, Ghanem ST, Dbaibo S. Review of meningococcal vaccines with updates on immunization in adults. Hum Vaccin Immunother. 2014;10:995-1007.

98. Velentgas P, Amato AA, Bohn RL, et al. Risk of Guillain-Barré syndrome after meningococcal conjugate vaccination. Pharmacoepidemiol Drug Saf. 2012;21:1350-8.

99. De Wals P, Deceuninck G, Boucher RM, Ouakki M. Risk of Guillain-Barrè syndrome following serogroup $C$ meningococcal conjugate vaccine in Quebec, Canada. Clin Infect Dis. 2008;46:e75-7.

100. Zheteyeva Y, Moro PL, Yue X, Broder K. Safety of meningococcal polysaccharide-protein conjugate vaccine in pregnancy: a review of the vaccine adverse event reporting system. Am J Obstet Gynecol. 2013;208:e1-6.

101. Toxnet [Internet]. Bethesda (MD): National Library of Medicine (US): [Last Revision Date Mar 10, 2015]. Meningococcal Vaccines; LactMed Record Number: 168 [about 3 p]. Available at: http://toxnet.nlm.nih.gov/cgi-bin/sis/search2/r? dbs+lactmed:@term+@DOCNO+168.

102. Anonymous. Meningococcal disease. Secondary attack rate and chemoprophylaxis in the United States, 1974. JAMA. 1976;235:261-5.

103. Zalmanovici Trestioreanu A, Fraser A, Gafter-Gvili A, Paul M, Leibovici L. Antibiotics for preventing meningococcal infections. Cochrane Database Syst Rev. 2011; CD004785.

104. Gardner P. Clinical practice. Prevention of meningococcal disease. $\mathrm{N}$ Engl J Med. 2006;355:1466-73.

105. Jorgensen JH, Crawford SA, Fiebelkorn KR. Susceptibility of Neisseria meningitidis to 16 antimicrobial agents and characterization of resistance mechanisms affecting some agents. J Clin Microbiol. 2005;43:3162-71.

106. Prasad K, Karlupia N. Prevention of bacterial meningitis: an overview of Cochrane systematic reviews. Respir Med. 2007;101:2037-43.

107. Schwentker FF, Gelman S, Long PH. The treatment of meningococcal meningitis with sulfonamide: preliminary report. JAMA. 1937;108:1407-8.

108. Shin SH, Kim KS. Treatment of bacterial meningitis: an update. Exp Opin Pharmacother. 2012;13:2189-206. 